\title{
Helium abundances in inner Galaxy planetary nebulae
}

\author{
Oscar Cavichia $^{1,2}$, Roberto D. D. Costa ${ }^{1}$, and Walter J. Maciel ${ }^{1}$ \\ ${ }^{1}$ IAG, University of São Paulo, 05508-900, São Paulo-SP, Brazil \\ ${ }^{2}$ email: cavichia@astro.iag.usp.br
}

\begin{abstract}
New helium abundances of planetary nebulae located towards the bulge of the Galaxy were derived, based on observations made at OPD (Brazil). We present accurate helium abundances for 56 PNe located towards the galactic bulge. The data show good agreement with other results in the literature, in the sense that the distribution of the abundances is similar to previous works. Furthermore, the radial helium gradient is extended towards the galactic center. The results show that no trend can be identified when comparing the internal gradient $(\mathrm{R} \leqslant 4$ $\mathrm{kpc})$ to the whole galactic disk.
\end{abstract}

Keywords. ISM: planetary nebulae: general; Galaxy: abundances, evolution

\section{Introduction}

Planetary nebulae (PNe) are an important tool to study the chemical abundances in the Galaxy. As an offspring of intermediate mass stars, their helium abundances suffer from contamination from the evolution of the progenitor star. Even when the contamination of the progenitor star is considered, the existence of a $\mathrm{He} / \mathrm{H}$ gradient in the galactic disk is very uncertain (see Maciel 2000). The derived $\mathrm{He} / \mathrm{H}$ gradients from $\mathrm{PNe}$ abundances are negligible, and can be written as $\mathrm{d}(\mathrm{He} / \mathrm{H}) / \mathrm{dR}=0.0000 \pm 0.0004$ (Maciel 2000).

Since bulge and disk may have formed in different ways such as represented by the disk inside-out formation model (Chiappini et al. 2001), or the model of multiple infalls onto the bulge (Costa et al. 2008), we would expect that these differences should appear in the abundance distributions of these structures. This way, in the present work we have extended the radial $\mathrm{He} / \mathrm{H}$ gradient of the disk $(\mathrm{R} \geqslant 4 \mathrm{kpc})$ towards the galactic center, in order to compare the $\mathrm{He} / \mathrm{H}$ gradient of both regions. This was done by using a new set of helium abundances of PNe located towards the galactic bulge derived by our group.

\section{Method}

Spectrophotometry observations in the optical domain were made at OPD observatory (Brazil) for a sample of 56 planetary nebulae located in the direction of the galactic bulge. The data were reduced following standard reduction procedures with the IRAF software (see Escudero et al. 2004). Details of the observation and data reduction procedures are described by Cavichia et al. (2009). The disk sample consists of 73 PNe compiled from our group (see for example Costa et al. 2004 and references therein).

In order to study the $\mathrm{He} / \mathrm{H}$ gradient, $\mathrm{PNe}$ distances were taken from Stanghellini et al. (2008). The heliocentric distances were converted to galactocentric distances adopting $\mathrm{R}_{\odot}=8 \mathrm{kpc}$. Additionally, new distances were derived for $46 \mathrm{PNe}$ using the statistical distance scale proposed by Stanghellini et al. (2008), and based on the $5 \mathrm{GHz}$ radio flux and optical angular diameter. In a first approach, no correction was considered for the contamination of the progenitor star evolution on the original helium abundance. 


\section{Results and discussion}

The He/H radial gradient is shown in Figure 1 . For $\mathrm{R} \leqslant 4 \mathrm{kpc}$ we addopted two different binnings in order to minimize the effect of the bin selection on the radial gradient.

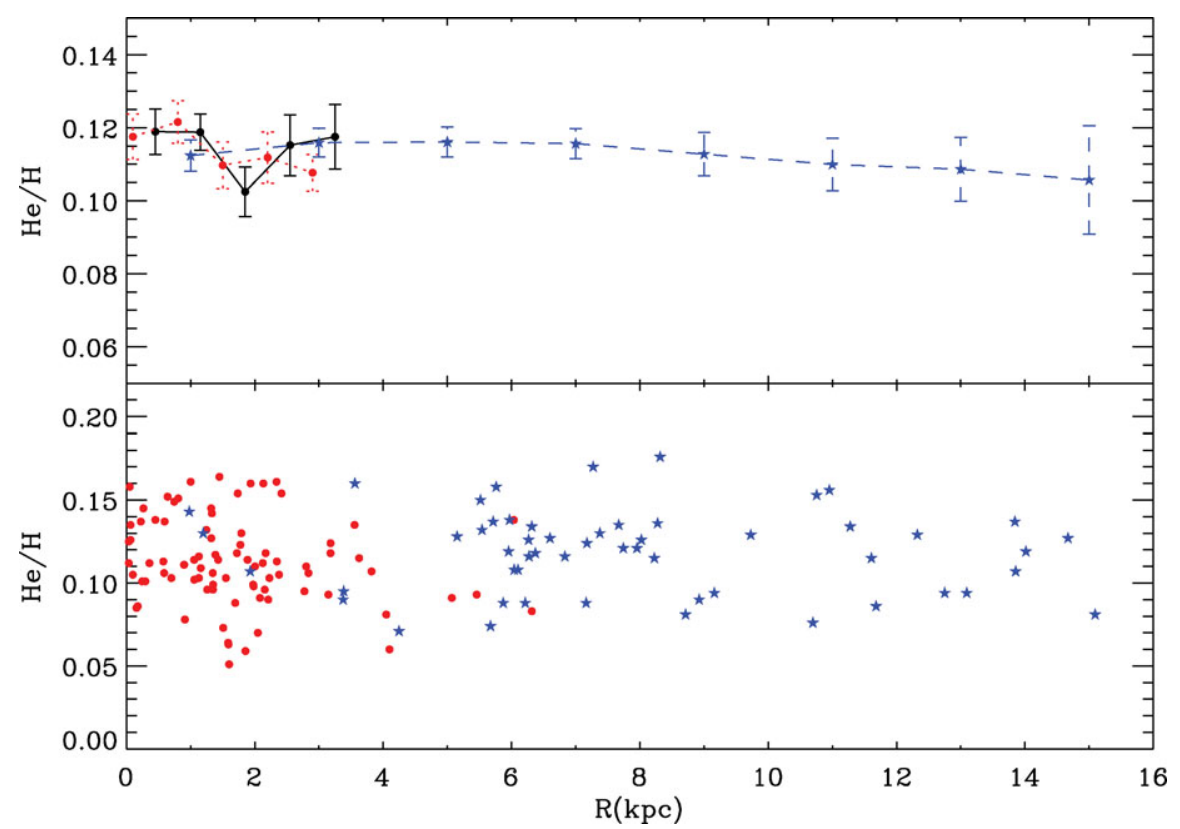

Figure 1. Top panel: the helium abundance as a function of the galactocentric distance for the bulge/inner disk sample (continuous and dotted lines represent the two different binnings) and for the disk sample (dashed line). For each bin the mean and the standard error of the mean (error bars) are given. Bottom panel: the radial distribution of helium abundances without considering mean abundances. Circles represent bulge/inner disk data and stars represent disk.

It can be seen in the figure that the $\mathrm{He} / \mathrm{H}$ gradient for $\mathrm{R} \leqslant 4 \mathrm{kpc}$ is negligible, as it is in the outer part of the disk. As a conclusion, more reliable abundances and distances, especially in the inner region of the Galaxy, are necessary in order to investigate the radial dependence of the helium abundance.

\section{References}

Cavichia, O., Costa, R. D. D., \& Maciel, W. J. 2009, Rev. Mexicana AyA, (Submitted)

Chiappini, C., Matteucci, F., \& Romano, D. 2001, ApJ, 554, 1044

Costa, R. D. D., Uchida, M. M. M., \& Maciel, W. J. 2004, A\&A, 423, 199

Costa, R. D. D., Maciel, W. J., \& Escudero, A. V. 2008, Baltic Astronomy, 17, 321

Escudero, A. V., Costa, R. D. D., \& Maciel, W. J. 2004, A\&ऽA, 414, 211

Maciel, W. J. 2000, in: L. da Silva, R. de Medeiros, \& M. Spite (eds.), The Light Elements and their Evolution, Proc. IAU Symposium No. 198, p. 204

Stanghellini, L., Shaw, R. A., \& Villaver, E. 2008, ApJ, 689, 194 